\title{
Nuevas formas de hacer política: Las amas de casa populares
}

Cecilia Blondet

En las siguientes páginas haremos algunos comentarios sobre los comedores populares y su potencialidad política como organización femenina de las amas de casa populares y como espacio común de aprendizaje democrático en la medida en que su práctica genera nuevas relaciones horizontales entre sus miembros. No desarrollaremos el tema de los comedores, ni mostraremos su funcionamiento ni las múltiples variedades que existen. Nos interesa particularmente comenzar a respondernos una pregunta muy general: ¿Por qué en los años 80 las amas de casa populares comienzan a crear organizaciones femeninas en las que se legitima su quehacer doméstico, se exige un reconocimiento a su aporte en la economía familiar y enfrenta desde ahí, como instancia pública de acción colectivamente a otras instituciones de la sociedad?

¿Es posible pensar en los comedores populares como organizaciones que fomentan un nuevo quehacer político a partir de una actividad doméstica como la cocina? De ser así, ¿podría pensarse en un movimiento de amas de casa que como tal, con una identidad política colectiva se articule al movimiento social? o más bien, ¿son una nueva respuesta de las mujeres como responsables del consumo familiar frente a la crisis que al cabo de un tiempo se dispersará como organización quedando en la memoria co- 
lectiva como otro momento más en el que fueron protagonistas?

Nos inclinamos a pensar en las primeras propuestas y a riesgo de ser consideradas optimistas e ilusas, ofrecemos algunos elementos que a nuestro juicio son importantes para enriquecer el debate: por un lado, la importancia de la familia como unidad de producción $\mathrm{y}$ reproducción de los sectores populares, $\mathrm{y}$ el rol de la mujer como productora de bienes domésticos y como administradora del consumo familiar. Y por otro, las experiencias históricas de participación femenina en el proceso de construcción de un espacio físico y social en la ciudad que ha sido posible por el esfuerzo individual y colectivo de los pobladores en general y de las mujeres en particular, forjando una nueva identidad como ciudadanos.

\section{LA FAMILIA COMO ESPACIO DE ACCION}

Las mujeres como responsables de la vida cotidiana, por el hecho de ser mujeres, asumen las tareas domésticas y reproductivas del hogar. Nadie cuestiona esto. Las mujeres lavan, cocinan, limpian y crian a los hijos mientras el hombre sale a la calle a obtener el ingreso familiar que permite que la mujer tenga qué cocinar, qué lavar y qué limpiar. El produce y ella administra. Este es el modelo básico de unidad doméstica que la división sexual del trabajo impone en la ciudad. A partir de esta propuesta, reconocemos-como "lo normal" el hecho de que la mujer trabaje en la casa, $y$ el hombre en la calle. Con esta asignación sexual a tareas y a responsabilidades, tenemos también definidos los espacios en los que se mueven los distintos miembros de una unidad doméstica, es decir, las mujeres con un reforzado rol reproductivo, doméstico y-en un espacio fundamentalmente privado, mientras el hombre asume el rol productivo en un espacio público. Con esta asignación, a su vez, estamos también delimitando los alcances de la unidad doméstica como el espacio privado de la reproducción fuera de los espacios de la producción.

Este modelo de unidad doméstica y de familia nuclear está explícitamente propuesto en la ideología dominante como la concepción de familia reconocida en la medida en que la familia es la unidad de reproducción de la fuerza de trabajo en la sociedad, por lo tanto es conceptualizada como una categoría incuestionable y ahistórica. Es "lo normal" y así lo refuerzan los medios masivos de comunicación. Pero este modelo tiene sentido si es que tenemos 
un Estado eficiente que cumple con facilitar los servicios públicos y la infraestructura necesaria para que las familias se desarrollen de acuerdo a estos principios y a este ordenamiento. Por otro lado, se requiere además de un mercado de trabajo lo suficientemente amplio como para permitir que la fuerza de trabajo se realice efectivamente en las instituciones sociales de la producción.

Paradójicamente, nuestra realidad está lejos de corresponder a lo descrito, aunque ideológicamente sigamos funcionando con tales concepciones y categorías respecto a la familia.

El hecho de tener una moneda devaluada y salarios reducidos que no alcanzan para satisfacer la canasta familiar, un aparato productivo estrangulado que produce caro y vende poco por consiguiente, un mercado de trabajo reducido y pobre y un Estado cada vez más ineficiente en su capacidad de respuesta a las exigencias de servicios públicos de la población, plantean una situación compleja que se define en la informalización de la sociedad obligando a los sectores populares a crear nuevas estrategias de sobrevivencia que se asientan fundamentalmente en la familia y los grupos de parientes y paisanos, transformando a su vez las familias en unidades de producción y reproducción simultáneamente. -

La unidad doméstica entonces, se va tornando en un espacio cada vez más importante y complejo de gestión multifamiliar que organiza su reproducción a partir de una economía familiar de ingresos agregados generado por sus miembros independientemente del sexo o la edad.

Las mujeres, por su parte, deben contribuir regularmente en el ingreso familiar, cuando no asumirlo en gran proporción ${ }^{1}$.

Una alternativa para ello, es que se incorporen al mercado de trabajo formal o informal como empleadas, obreras, ambulantes, destajeras entre otras, pero con las reconocidas limitaciones debido a la escasa formación que poseen, que las lleva por lo general a retomar empleos domésticos con un carácter de eventualidad $^{2}$. Otra posibilidad es que las mujeres pobladoras, en el propio barrio, encuentren formas alternativas de solución al problema de la alimentación familiar, que en última instancia es el problema más agudo de la vida cotidiana. El hambre, si bien afecta a todos los miembros, su atención es de responsabilidad en primer lugar del ama de casa. Para esto, si las pobladoras tienen suerte y una cierta calificación como costureras o tejedoras, podrán contactarse con intermediarios para realizar trabajos a domicilio como 
destajeras; si no, siempre queda la alternativa de suscribirse a los programas de apoyo alimentario sean de la parroquia, del gobierno o de. otras iglesias, aunque estos programas sólo ofrezcan una solución parcial al problema.

Todas las alternativas descritas tienen además de la limitación que se basa en la calificación o no de las mujeres, la limitación del momento del ciclo de vida familiar en que las toma la crisis y las posibilidades de organizar las tareas de la unidad doméstica para poder acceder a trabajos extradomésticos. Es decir, no todas las mujeres aunque sean calificadas para trabajos especializados pueden salir a trabajar si tienen muchos hijos y éstos están chiquitos. En cambio si los hijos son más grandes, se pueden cuidar entre ellos, van al colegio y dejan un tiempo libre, colaboran en las tareas domésticas compartiendo con la madre el trabajo o salen a trabajar ayudando en el ingreso familiar. Todas estas posibilidades (que pueden o no darse dependiendo de múltiples factores) apuntan a liberar parte del tiempo de trabajo doméstico, que la mujer puede dedicar luego a otras actividades.

Asimismo, es posible considerar las relaciones de ayuda mutua y de parentesco espiritual que entablan las mujeres en los barrios como redes de solidaridad inicial y primaria, también como una forma de liberar tiempo doméstico para disponerlo a trabajos extradomésticos. Pero si bien estas relaciones existen y cumplen un rol muy importante sobre todo en los primeros momentos de ocupación del lote, luego se van diluyendo en la medida en que cada familia se va concentrando en su propia construcción. Como en este proceso todos los pobladores enfrentan una serie de problemas, las posibilidades de ayuda entre ellos por lo general se reducen a situaciones extremas. Prima el individualismo sobre la solidaridad inicial. Por lo tanto para pensar en nuevas organizaciones es preciso construir una nueva red de solidaridades que nuclee en este caso a las mujeres y que les permita reconocer un problema común y la importancia de resolverlo colectivamente, sobre el cual tejer la organización. Debe existir un eje de preocupación que recoja la atomización y convierta los casos individuales en asunto colectivo.

Pero si bien las relaciones de ayuda mutua vecinal o de parentesco espiritual no funcionan con la eficiencia inicial, los grupos de parientes pueden llegar a constituirse en alternativa de organización micro-comunal importante, reubicando a las mujeres 
en una compleja organización multifamiliar ${ }^{3}$. En cualquier caso, las mujeres pobladoras amas de casa en momentos de crisis, con un creciente deterioro de las relaciones sociales y ante la precariedad de la vida cotidiana que se traduce en el hambre familiar, deben actuar, asumiendo nuevamente roles protagónicos en la escena social urbana. Si lo hacen de manera individual o si se van creando estas nuevas redes de solidaridad, con todos los conflictos propios de la vida en la miseria es tema de discusión. Lo que no es tema de discusión es que las mujeres están participando activamente en la consecución de ingresos para la economía familiar, sin lugar a dudas, y que esta participación comienza a proponer una transformación de la unidad doméstica y de la ubicación de la mujer en ella.

\section{LA HISTORIA DE PARTICIPACION Y EL APRENDIZAJE DE LA CIUDADANIA}

No queremos referirnos a la historia de construcción del barrio sino más bien a cómo esta historia de participación y en concreto la necesidad de construir un espacio físico en la ciudad ha demandado un extraordinario esfuerzo de movilización y lucha, convirtiendo esta experiencia en un aprendizaje sin par de prácticas políticas, que posteriormente constituirán una referencia importante para la creación de nuevas organizaciones.

Por eso, resaltamos sólo un momento de la historia que se refiere a los primeros tiempos de la ocupación y que muestra la importancia de la participación femenina en acciones colectivas para conseguir objetivos fundamentalmente individuales y familiares.

En primer lugar este período exige una permanente confrontación con diversas entidades gubernamentales para consolidar su posesión: los lotes no se asignan, se lucha por ellos, los servicios de urbanización no se donan, tienen que exigir y trabajar para conseguirlos, los colegios y postas médidas no aparecen por ley, tienen que organizarse, reclamar para su asignación y finalmente hacerlos ellos mismos.

En segundo lugar y viendo el aspecto individual, la participación de las mujeres pobladoras en estos años está estrechamente vinculada al ciclo de vida familiar y al proyecto de construcción de la unidad doméstica que en última instancia es el eje del enraizamiento urbano. Por ello su presencia en las primeras moviliza- 
ciones vecinales por la consecusión de servicios para el barrio es masiva pero eventual e inorgánica. El motivo de esta participación es la exigencia al Estado de seguridades para la consolidación del lote y de la unidad doméstica y no conlleva necesariamente una orientación hacia el cambio social, ni tiene un objetivo político definido. Esta característica explica por qué las mujeres no aspiran ni detentan el poder en las organizaciones vecinales.

Independientemente del grado de conciencia de clase y de género que tengan las mujeres pobladoras $\mathrm{y}$ del logro concreto que obtengan de su participación en las movilizaciones, el solo hecho de participar es importante como parte de un aprendizaje político que las enfrenta por primera vez a las instituciones de la sociedad. Por eso, la práctica de formas democráticas de participación, aún cuando se realizan para lograr objetivos muy primarios e individuales, desde lo doméstico, irán permeabilizando,creemos, tanto a hombres como mujeres hacia una transformación de la unidad doméstica.

Luego de los primeros años de construcción barrial, las organizaciones vecinales en las que participan las mujeres como apoyo en las movilizaciones, o como impulsoras del trabajo en faena para el propio barrio, comienzan a relajarse y las mujeres se concentran en el espacio familiar, que no es necesariamente un espacio estrictamente doméstico en tanto deben realizar múltiples trabajos para su consolidación.

A lo largo de estos años, surgen entonces los primeros clubes de madres, que son instancias locales de reunión femenina y que nacen como una forma de institucionalización de la ayuda mutua entre mujeres en la primera época pero con una clara influencia de entidades asistencialistas gubernamentales o cristianas que desde mediadog-de los 70 adquieren un rol protagónico.

Estas entidades asistencialistas las habilitan de alimentos donados a cambio de trabajos colectivos, charlas evangélicas o cursillos, imponiendo un tipo bastante clientelista de organización tanto en relación con las entidades asistencialistas como entre las socias. Por eso si bien, en un primer momento, la vida orgánica de los clubes es fluctuante y se caracteriza por tener una relación posesiva, arbitraria y caciquista entre la presidenta y las socias, en momentos de crisis, las mujeres pobladoras más jóvenes comienzan a cuestionar este tipo de organización, a demandar una utilidad inmediata de la participación y de alguna manera replantean el sentido de los clubes. Se imprime una nueva dinámica entre di- 
rigentes y socias y a través de estas instancias, se busca responder a la demanda de contribución en la economía familiar a través del reparto de alimentos que estos ofrecen. Asimismo, buscan en los cursos de capacitàción, conocimientos prácticos que reviertan rápidamente en técnicas de producción artesanal para hacer chompas, muñecos o tortas que luego pueden vender.

No obstante, la relación entre dirigentes y entidades asistencialistas, sigue signada por un clientelismo que comienza a plantear nuevas condiciones, que se va reformulando, pues son concientes de que no se pueden "liberar" de su aporte por la necesidad de contar con los alimentos como apoyo para la cocina diaria.

Tanto la participación en movilizaciones, la organización del trabajo en faena para la construcción barrial como la asistencia a los clubes de madres y las experiencias de algunas mujeres como esposas de obreros en la olla común de la toma de fábricas son antecedentes de los comedores populares, que a su vez, recogen la urgencia de las amas de casa de responder al hambre familiar actual.

\section{LOS COMEDORES FAMILIARES, UNĀ RESPUESTA COMPLEJA}

Retomando en síntesis algunas ideas de las páginas anteriores tenemos que el momento actual entre otras cosas, se caracteriza por la crisis y el hambre popular, tenemos también muy clara la responsabilidad familiar frente al problema debido a una ausencia significativa del aparato estatal y finalmente, tenemos que dentro de la familia, la importancia que la mujer asume en la solución del problema, es central, bien sea consiguiendo ingresos considerables o administrando y complementando la economía familiar mediante la participación en clubes o comedores populares.

Entonces surge nuevamente la pregunta: ¿Qué son los comedores populares? ¿Son estos una nueva organización que ofrece hasta cierto punto una solución parcial al problema alimentario, especialmente en el caso de las mujeres amas de casa que no pueden salir a trabajar fuera del barrio? ¿A quiénes reúne? ¿Si es más que eso, por qué es así?

No-conocemos todos los tipos de comedores que existen y sabemos que hay gran variedad: desde los parroquiales, los politicos (del APRA, AP, IU), los autosostenidos, los que reciben apo- 
yo de iglesias ecuménicas, o los familiares ${ }^{4}$. En este trabajo, sólo nos referiremos a los familiares porque creemos que expresan más claramente el objetivo que estamos observando en estas organizaciones, el conflicto de llevarlas adelante y su potencialidad política como organización de amas de casa populares.

Quizá la única condición para participar en estos comedores es sentir hambre. Quien no lo siente, dicen ellas no participa pues es tan grande el esfuerzo que demandan que no se justifica sin el apremio.

Se organizan entre doce y quince familias y dan una cuota de dinero, es decir, entre doce y quince mujeres que tienen detrás una familia con hambre. Reuniendo ollas, hornillas y cucharones, escogen la casa más aparente entre las socias para instalar el comedor, que más directamente es una cocina familiar. Establecen un ordenado sistema de turnos para realizar colectivamente todas las tareas y luego se distribuyen: unas compran los alimentos y otras cocinan, limpian y reparten. Al mediodía están las raciones listas en las ollas de cada una y ellas mismas o sus hijos pasan a recogerlas. El consumo es familiar y está prohibido vender las raciones extras que queden para evitar la especulación, aún a costa de la posibilidad de capitalización a partir de la venta del alimento excedente.

Veamos ahora los problemas. El más serio es lograr que todas cumplan con su tarea y que no abandonen a la mitad pues rompen el orden y la lógica de funcionamiento. Otros problemas como el sabor de la comida, el cuidado de los enseres, la cantidad que cada equipo logra producir o la puntualidad, constituyen roces cotidianos que se van superando o no conforme se va constituyendo el grupo.

Un-problema de fondo que tiene que ver con el límite económico impuesto por el reducido ingreso familiar, más que por problemas en la organización en sí, es el de la composición nutricional de las raciones. Distan mucho de ser dietas balanceadas y más bien los menús apuntan a llenar los estómagos de la familia antes que a reponer eficientemente las energías de la jornada. Este es un problema reconocido por ellas y que expresa los límites de la organización en la pobreza.

Otro problema grande es la competencia entre los diferentes tipos de comedores, especialmente entre estos que pueden considerarse como el germen de un nuevo tipo de organización que se caracteriza por la autonomía y los comedores impulsados por par- 
tidos políticos, sobre todo las cocinas de AP y del APRA que nacen y se instalan sustentados en un explícito objetivo clientelista. No es que creamos que el hecho de entregar grandes ollas, ofrecer locales apropiados y subvencionar las raciones es de por sí negativo. En otras palabras, que pensemos que a mayor dificultad en el proceso de constitución, mayor éxito en la organización. El problema se presenta cuando, como en los comedores administrados por partidos políticos, no se sitúa el objetivo de la organización en la necesidad de hacerle frente al hambre familiar de manera colectiva, sino más bien se utiliza el hambre familiar y el dinero partidario para conseguir más votos en las próximas elecciones, dejando de lado el sentido mismo de la organización. Esto no quiere decir que en algunos casos los comedores impulsados por partidos politicos no pueden devenir en organizaciones cada vez más autónomas y que en la práctica cotidiana vayan desprendiéndose del patrón. Los comedores también pueden ser una plataforma. El problema se agudiza cuando crece la necesidad y disminuyen las posibilidades de satisfacerlas por sí solas.

No obstante lo mencionado, hay muchas razones que animan a las amas de casa a continuar en esta experiencia de comedores familiares. El primero y más evidente es la constatación de la ventaja de la utilización de una economía de escala que abarata el costo de producción alimentaria familiar. Si no se logra este objetivo eficientemente, el grupo fracasa irremediablemente. Nuevamente encontramos la organización colectiva para solucionar problemas familiares, es decir, al igual que en los primeros momentos de la ocupación, los pobladores recurren a lo colectivo para propósitos individuales y en esta práctica desde lo cotidiano, va forjando un nuevo tipo de organización más autónoma y política.

Una segunda ventaja de los comedores es la de liberar parte del tiempo que la mujer dedica a tareas domésticas facilitando por un lado el acceso de las mujeres a otros trabajos eventuales que incrementen el ingreso familiar, o bien, abriendo nuevas posibilidades de participación en cursos de capacitación, en actividades dirigenciales $o$ en otro tipo de organizaciones comunales $y$ vecinales.

La tercera ventaja es la revitalización de redes de solidaridad intervecinal. Esto es importante especialmente si tenemos en cuenta que tanto por efecto de la crisis como por la dinámica misma del ciclo de vida familiar, las relaciones entre los pobladores no son siempre fluidas ni organizadas y que más bien tienden a 
la atomización y al conflicto, sobre todo en situaciones de miseria como la que se vive actualmente. El hecho de ponerse en contacto para organizar y llevar adelante el comedor, requiere de un acercamiento especial entre las participantes que, superando el conflicto inicial, va creando un sentido de grupo y un espacio importante de socialización de problemas personales. El comunicarlos y reconocer situaciones comunes de violencia, agresión y subordinación en las relaciones intrafamiliares es un acto importante de reconocimiento como personas. Por ejemplo, en casos de violencia conyugal, se han dado casos en los que las vecinas han intercedido por la señora golpeada, a pesar de amenazas del marido, los consejos del cura para que no se metan y la desidia de la Guardia Civil para tomar cartas en el asunto y controlar la situación. De otra manera, pero también dentro de las formas solidarias que surgen en los comedores, encontramos que se estudian los casos en los que no se puede dar la cuota e inclusive se desarrollan mecanismos de reemplazo de turnos entre ellas. Con esto queremos decir cómo en una situación de conflicto casi permanente que emana de la precariedad de la vida cotidiana y de la incapacidad casi total de prever el día siguiente, los comedores pueden desarrollar nuevas y aún frágiles formas de solidaridad básica, germen para las nuevas organizaciones que se van creando.

Finalmente creemos que el aprendizaje de prácticas democráticas interpersonales es un paso importante para combatir el clientelismo. El hecho de someterse a una disciplina de trabajo horizontal, a turnos y horarios y especialmente el tener relación como grupo con otras instituciones de la sociedad como el Municipio, la parroquia o la Federación del barrio son indudablemente parte de este aprendizaje del quehacer público. De otro lado, el hecho de ser autosostenidos, habla ya de un grado de autonomia de las entidades asistenciales y los programas de apoyo alimentario que resulta sugerente; aunque esta autonomia no necesariamente sea tan real como aparece, pues por lo general entre telones, se encuentra la presencia discreta de un centro de Educación popular, de un grupo de cristianos de base, o instituciones que son también conscientes de la dificultad de esta tarea en este momento y por lo tanto, de la necesidad de una asesoría que la refuerce.

En consecuencia, creemos que los comedores actualmente son organizaciones básicamente defensivas frente a situaciones de 
crisis y ante el reconocimiento de la necesidad de actuar colectivamente.

Las reivindicaciones que levantan se refieren por lo general a problemas vinculados al quehacer doméstico que las reúne. Sin embargo, el hecho de hacerlos colectivamente, tornando el problema personal en un problema social es importante, pues comienzan a reconocer los límites de la organización en la pobreza y por lo tanto la posibilidad de utilizar los propios comedores como instancias que vayan más allá de la respuesta al problema alimentario y planteen un cuestionamiento al Estado y a la distribución del ingreso.

\section{LOS COMEDORES COMO ORGANIZACIONES POTENCIA LMENTE POLITICAS}

Por lo dicho anteriormente y a manera de conclusión, planteamos algunos puntos sobre la importancia de los comedores familiares y retomamos la segunda pregunta que planteamos al inicio en cuanto a la potencialidad política de estas instituciones: ¿Los comedores desarrollan una nueva forma de hacer política?

Los comedores familiares transfieren el quehacer doméstico que las mujeres realizan en el ámbito privado, al ámbito público y colectivo, mostrando la estrecha relación e influencia que lo privado ejerce en lo público y viceversa.

$\mathrm{Al}$ hacer públicas y visibles las tareas domésticas se comienza a ampliar en la práctica, los estrechos márgenes reproductivos de la unidad doméstica como unidad de consumo, como sucede con las estrategias multifamiliares, pero a diferencia de éstas, los comedores legitiman el carácter colectivo y público de la organización. Esta característica, a su vez abre la posibilidad de replantear la ubicación de la mujer en la unidad doméstica, transformando roles y funciones tradicionalmente asignadas a ella por la división sexual del trabajo y la ideología dominante.

Si bien en los comedores se evidencia la interrelación entre los espacios público y privado, su ámbito de acción es básicamente local y por lo general circunscrito a la solución del problema alimentario. Sin embargo, se están creando nuevas instancias organizativas que comienzan a nuclear a las dirigentas alrededor de planteamientos que se proponen objetivos que van más allá de la cocina comunal. Organizaciones como la Federación de Mujeres de Villa El Salvador por ejemplo, están proponiendo el control de 
la donación y el reparto de alimentos, antes efectuado por las instituciones asistenciales, redefiniendo las relaciones verticales y clientelistas impuestas por las instituciones que los donan. De esta manera, a partir de la experiencia de los comedores, se intenta generalizar el aprendizaje de nuevas formas democráticas y horizontales en las relaciones sociales.

De igual forma, pero en aspectos vinculados propiamente a la gestión administrativa de los comedores, surge ya en algunas mujeres dirigentas, la exigencia al municipio de abaratar el precio de los alimentos y de los insumos en general que abastecen los comedores.

Indudablemente, no es posible aún generalizar este comportamiento directamente político a todos los comedores, ni mucho menos a todas las mujeres que participan en estos. Lo que sí es posible, es pensar a partir de las manifestaciones mencionadas, en estas organizaciones como instituciones potencialmente políticas en las que las mujeres se definen como actores colectivos de la escena social, en una nueva forma de hacer política desde el quehacer doméstico. La práctica cotidiana del trabajo colectivo y la experiencia organizativa que conlleva, está permitiendo que las mujeres comiencen a demandar en una actitud fiscalizadora, dignidad y mejores condiciones de vida para ella y su familia, exigiendo así, la ampliación de los derechos de ciudadanía. 
NOTAS

${ }^{1}$ Sin embargo por lo general se mantiene la concepción del trabajo femenino como trabajo complementario y subordinado al ingreso del jefe de familia.

${ }^{2}$ Las mujeres populares, especialmente las migrantes poseen por lo general una baja calificación debido al acceso restringido a los centros de educación y cuentan únicamente con el conocimiento y la habilidad del trabajo doméstico, que es parte de su socialización familiar y social. Por esto, su participación en el mercado laboral y en empleos remunerados en la mayoría de los casos tiene esta connotación. Su especificidad como mujeres trabajadoras, está definido por su rol doméstico y no por su potencial rol productivo. Los trabajos que absorben mayor canti- dad de mano de obra femenina en la industria y el comercio presentan esta característica, (ver Barrig, Chueca, Yáñez, 1985). Las empleadas domésticas, finalmente, son la expresión más clara de esta relación femenino-doméstica.

3 Ver Golte y Adams, "Lus caballos de Troya ... .", 1984.

${ }^{4}$ Se denominan así al tipo de comedores que surgen en algunos barrios populares y que juntan grupos de un número determinado de familias. Por lo general, están siendo impulsados por dirigentes con experiencia anterior en el movimiento barrial o por el propio municipio. En este caso, trataremos sobre los comedores familiares de Villa El Salvador. 


\section{BIBLIOGR AFIA}

BARNECHEA, María Mercedes y DELPINO

1985 "Comedores Populares: una mirada desde adentro". En Materiales para la Comunicación Popular No. 6 (en prensa).

BARRIG, M., M. CHUECA y A. M. YANEZ

1985 Anzuelo sin carnada: obreras en la industria de conserva de pescado. Mosca Azul, Lima.

FEIJOO, Maria del Carmen

1983 Buscando un techo: Familia y vivienda popular. Estudios CEDES, Buenos Aires.

HUAMAN, Josefina

1985 "Comedores populares y estrategia de supervivencia" En Tarea, No. 12, julio 1985.

JELIN, Elizabeth

1978

La mujer y el mercado de trabajo urbano. Estudios CEDES, Buenos Aires.

1983 Las relaciones sociales del consumo: el caso de unidades domésticas de sectores populares. The Population Council, Documento de Trabajo No. 14, México.

JELIN, Elizabeth y FEIJOO, M.

1980

Trabajo y familia en el ciclo de vida femenino: el caso de los sectores populares de Buenos Aires. Estudios CEDES, Buenos Aires.

SARA LAFOSSE, Violeta

1984 Los comedores comunales en los barrios populares de la ciudad de Lima. Informe final de investigación, Grupo de Trabajo: "Servicios Urbanos y Mujeres de bajos ingresos". Lima, mimeo. 